\title{
A NOTE ON THE BOCKSTEIN OPERATOR
}

\author{
HANS SAMELSON ${ }^{1}$
}

1. Introduction. The Stiefel-Whitney classes $w_{1}, \cdots, w_{n}$ of an $n$ plane bundle $\xi$ over a space $X$ are certain well-defined elements of $H^{*}\left(X ; Z_{2}\right)$ (singular cohomology). For the effect of the Steenrod squares on them one has the Wu formulae $S q^{i} w_{j}=\sum_{0}^{i}\left(\frac{j-i+t-1}{t}\right) w_{i-t} w_{j+t}$ [2]. In particular, since $S q^{1}=\beta_{2}$ (the Bockstein operator for the coefficient sequence $0 \rightarrow Z_{2} \rightarrow Z_{4} \rightarrow Z_{2} \rightarrow 0$ ) one has

$$
\beta_{2} w_{2 i}=w_{2 i+1}+w_{1} w_{2 i} \quad\left(\text { here } w_{j}=0 \text { for } j>n\right) .
$$

The classes $w_{2 i+1}$, for $2 i+1 \leqq n$, are $Z_{2}$-reductions of classes that we shall write as $\tilde{w}_{2 i+1}$, defined over a local coefficient system $Z$ whose group is $Z$ (the integers), the local system being determined by the orientation of the fiber, $[1$, p. 195] (incidentally, this also holds for $w_{n}$, in case of even $n$ ). With $\widetilde{\beta}$ denoting the Bockstein operator for the sequence $0 \rightarrow Z \rightarrow Z \rightarrow Z_{2} \rightarrow 0$, one has then, according to [1], the formulae

$$
\tilde{\beta} w_{2 i}=\tilde{w}_{2 i+1} \quad\left(\text { again } \tilde{w}_{j}=0 \text { for } j>n\right),
$$

and consequently, writing $\rho_{2}$ for reduction $\bmod 2$ and $\widetilde{\beta}_{2}$ for $\rho_{2} \circ \widetilde{\beta}$,

$$
\tilde{\beta}_{2} w_{2 i}=w_{2 i+1}, \quad \text { for } 0<2 i \leqq n ;
$$

for $i=0$ the situation is a little different, cf. $\$ 4$ below.

Our aim is to connect the two formulae (I) and (II), by means of the theorem in $\$ 3$.

2. Pairing of coefficient sequences. Let $0 \rightarrow A^{\prime} \rightarrow A \rightarrow A^{\prime \prime} \rightarrow 0,0 \rightarrow B^{\prime}$ $\rightarrow B \rightarrow B^{\prime \prime} \rightarrow 0$ be two exact sequences of coefficient groups (or local systems for cohomology in $X$ ) ; let $\alpha, \beta$ be the corresponding Bockstein maps. There is the usual exact sequence $0 \rightarrow D \rightarrow A \otimes B \rightarrow A^{\prime \prime} \otimes B^{\prime \prime} \rightarrow 0$, where $D$ is the natural image of $A^{\prime} \otimes B \oplus A \otimes B^{\prime}$ in $A \otimes B$. We can "divide" by the natural image of $A^{\prime} \otimes B^{\prime}$ in $A \otimes B$, and get the exact sequence (defining $C$ as image of $D$ )

$$
0 \rightarrow C \rightarrow A \otimes B / i\left(A^{\prime} \otimes B^{\prime}\right) \rightarrow A^{\prime \prime} \otimes B^{\prime \prime} \rightarrow 0,
$$

which we call the product sequence; let $\gamma$ be the associated Bockstein

Presented to the Society, November 19, 1963 under the title On Poincare duality; received by the editors November 12, 1962 and, in revised form, January 31, 1963.

1 Prepared with support from National Science Foundation Grant G-20301. 
operator. Let $k: A^{\prime} \otimes B^{\prime} \rightarrow A^{\prime} \otimes B \oplus A \otimes B^{\prime}$ be defined by $k\left(a^{\prime} \otimes b^{\prime}\right)$ $=\left(a^{\prime} \otimes b^{\prime},-a^{\prime} \otimes b^{\prime}\right)$. One verifies that the maps $A \rightarrow A^{\prime \prime}, B \rightarrow B^{\prime \prime}$ give rise to the following commutative diagram:

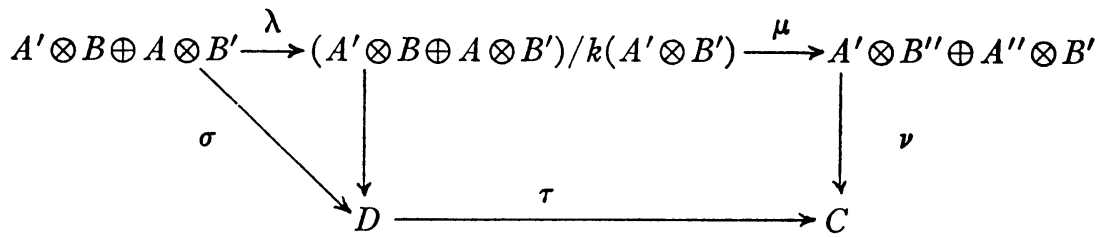

Let now $u \in H^{s}\left(X ; A^{\prime \prime}\right), v \in H^{t}\left(X ; B^{\prime \prime}\right)$ be given. We regard $\alpha u \cdot v+(-1)^{s} u \cdot \beta v$ as lying in $H^{s+t+1}\left(X ; A^{\prime} \otimes B^{\prime \prime} \oplus A^{\prime \prime} \otimes B^{\prime}\right)$, and $u \cdot v$ as lying in $H^{s+t}\left(X ; A^{\prime \prime} \otimes B^{\prime \prime}\right)$. We write $\lambda$, resp. $\lambda_{*}$, for the coefficient maps, defined by $\lambda$, on cochains, resp. cohomology.

Proposition. (a) $\gamma(u \cdot v)=\nu_{*}\left(\alpha u \cdot v+(-1)^{s} u \cdot \beta v\right)$; (b) $\alpha u \cdot v$ $+(1-)^{s} u \cdot \beta v \in \operatorname{im} \mu_{*}$.

Proof. Take cochains $x, y$ representing $u, v$; "pull back" to cochains $\bar{x}, \bar{y}$ with coefficients in $A, B$. Then $d(\bar{x} \cdot \bar{y})$, with coefficients in $A \otimes B$, pulls back to $D$; similarly $d \bar{x} \cdot \bar{y}+(-1)^{s} \bar{x} \cdot d \bar{y}$ has coefficients in $A^{\prime} \otimes B \oplus A \otimes B^{\prime}$; their $\tau_{*^{-}}$, resp. $\tau_{*} \circ \sigma_{*^{-}}$-images are equal and represent $\gamma(u \cdot v)$. One checks that $\lambda_{*}\left(d \bar{x} \cdot \bar{y}+(-1)^{s} \bar{x} \cdot d \bar{y}\right)$ is a cocycle (the two $A^{\prime} \otimes B^{\prime}$ have become equal). Applying $\mu_{*}$ to its cohomology class, we get part (b) of the proposition, and continuing with $\nu_{*}$ we get part (a).

3. The twisted Bockstein operator. Suppose $X$ is pathwise connected and has a base point. Let $p$ be any prime, and let $w$ be an element, $\neq 0$, of $H^{1}\left(X ; Z_{p}\right)$. By the universal coefficient theorem, $w$ defines and is in fact equivalent to a homomorphism of $H_{1}(X)$ (over $Z$ ) onto $Z_{p}$. Composing with the Hurewicz map we get a homomorphism, $w$, of $\pi_{1}(X)$ onto $Z_{p}$. Now the group $Z_{p^{2}}$ has an automorphism of order $p$, sending 1 into $p+1$, that leaves the subgroup $Z_{p}$ and the corresponding quotient group elementwise fixed. We regard this as an action of $Z_{p}$ on the sequence $0 \rightarrow Z_{p} \rightarrow Z_{p^{2}} \rightarrow Z_{p} \rightarrow 0$. Composing with $w$, we get a left action of $\pi_{1}(X)$ on this sequence, and thus a sequence $0 \rightarrow Z_{p} \rightarrow Z_{p^{2}} \rightarrow Z_{p} \rightarrow 0$ of local systems of coefficients in $X$, where the end terms are constant and the middle term has group $Z_{p^{2}}$. Write $\beta^{w}$ for the Bockstein operator of this sequence; also write $\beta_{p}$ for the Bockstein map of the constant sequence $0 \rightarrow Z_{p} \rightarrow Z_{p^{2}} \rightarrow Z_{p} \rightarrow 0$. For $w=0$ define $\beta^{0}=\beta_{p}$.

Theorem. For any $v \in H^{*}\left(X ; Z_{p}\right)$ we have

$$
\beta^{w}(v)=\beta_{p}(v)+w \cdot v \text {. }
$$


Proof. The case $w=0$ is trivial. Now let us take $w \neq 0$. First take $v=1$, the unit of $H^{*}\left(X ; Z_{p}\right)$. Here the result to be proved is $\beta^{w}(1)=w$, since $\beta_{p}(1)=0$. It is sufficient to consider 1 -simplices with both end points at the base point (use the 1st Eilenberg subcomplex of the singular complex). But then the relation to be proved is practically a tautology; note $(p+1)^{i} \equiv p \cdot i+1\left(\bmod p^{2}\right)$.

Second, we apply the considerations of $\$ 2$ to the sequence $0 \rightarrow Z_{p}$ $\rightarrow Z_{p^{2}} \rightarrow Z_{p} \rightarrow 0$ above and the constant sequence $0 \rightarrow Z_{p} \rightarrow Z_{p^{2}} \rightarrow Z_{p} \rightarrow 0$. The general formulae simplify a good deal: $i\left(A^{\prime} \otimes B^{\prime}\right)$ is 0 , so that $D=C ; k\left(A^{\prime} \otimes B^{\prime}\right)$ is also 0 ; the product sequence is isomorphic to the first given sequence; so that $\gamma=\alpha\left(=\beta^{w}\right)$; both terms $A^{\prime} \otimes B^{\prime \prime}$ and $A^{\prime \prime} \otimes B^{\prime}$ are of the form $Z_{p} \otimes Z_{p} \approx Z_{p}$ and map onto $C \approx Z_{p}$ under $\nu$. Part (a) of the proposition can then be interpreted as saying

$$
\beta^{w}(u \cdot v)=\beta^{w} u \cdot v+(-1) \cdot u \cdot \beta_{p} v,
$$

where all coefficients are $Z_{p}$ and the product is the usual U-product over $Z_{p}$.

We now take $u=1$ and get $\beta^{w}(v)=\beta^{w}(1 \cdot v)=\beta^{w}(1) \cdot v+\beta_{p} v=w \cdot v$ $+\beta_{p} v$, thus proving the theorem.

As an example, with $p=2$, take infinite real projective space with $H^{*}\left(P R^{\infty} ; Z_{2}\right)=Z_{2}[w]$. One finds that $\beta^{w}$ vanishes on the odd powers of $w$, and $\beta_{2}$ on the even powers.

We now apply our theorem to the situation of $\$ 1$, with $p=2$. We have of course $\widetilde{\beta}_{2}=\beta^{w_{1}}$, and so (I) and (II) are equivalent by the theorem (note $+=-$ here).

4. The case $i=0$. For the Stiefel-Whitney classes in dimensions 0 and 1 , Steenrod has the formula

$$
\widetilde{\beta} 1=\tilde{w}_{1}
$$

where one has to interpret 1 as the integral unit class, and one uses for the construction of $\tilde{\beta}$ the sequence $0 \rightarrow Z \rightarrow B \rightarrow Z \rightarrow 0$, where the group of $B$ is $Z \oplus Z$, and $\pi_{1}(X)$ acts by interchanging the two terms. Reducing $\bmod 2$ we get the sequence $0 \rightarrow Z_{2} \rightarrow U \rightarrow Z_{2} \rightarrow 0$, where the group of $\mathcal{V}$ is $Z_{2} \oplus Z_{2}$, and the relation

$$
\rho_{2} \circ \widetilde{\beta}(1)=w_{1},
$$

where now 1 is over $Z_{2}$. To "explain" this we consider the situation analogous to that of $\S 3: w_{1} \neq 0$, in $H^{1}\left(X ; Z_{2}\right) ; \pi_{1}(X)$ acting on $Z_{2} \oplus Z_{2}$ "according to $w$ "; $\beta^{\prime}$ the corresponding Bockstein operator. One verifies as in $\$ 3$ again $\beta^{\prime}(1)=w$; and $\rho_{2} \circ \widetilde{\beta}$ in $\left(I_{0}\right)$ is just the $\beta^{\prime}$ for $w=w_{1}$. 
AcKnowledgment. The author's original version of $\beta^{w}$ was limited to the case $p=2$. The referee suggested the extension to arbitrary $p$; he also suggested to expand the original proof of the theorem to the proposition on pairing in $\$ 2$.

\section{BIBLIOGRAPHY}

1. N. E. Steenrod, The topology of fibre bundles, Princeton Univ. Press, Princeton, N. J., 1951.

2. W. T. Wu, Les i-carrés dans une varieté grassmannienne, C. R. Acad. Sci. Paris 230 (1950), 918-920.

STANFORD UNIVERSITY 